\title{
Phase Transitions in Fluid State of Systems of Purely Repulsive Potentials
}

\author{
Shiqi Zhou ${ }^{*}, 1$, Hui $\mathrm{Xu}^{1}$ and Baoquan Zhang ${ }^{2}$ \\ ${ }^{1}$ School of Physics Science and Technology, Central South University, Changsha, Hunan, 410083, China \\ ${ }^{2}$ School of Chemical Engineering and Technology, Tianjin University, Tianjin, 300072, China
}

\begin{abstract}
Phase behaviors in fluid state of systems of purely repulsive potentials (PRPs) are investigated with a recently proposed 3rd-order thermodynamic perturbation theory (TPT) (Phys. Rev. E. 2006, 74, 031119). It is found that a usual gas-liquid transition (GLT) always happen to several investigate PRPs, whose perturbation part as a function of particle separation holds a discontinuous point, or an indifferentiable point, or is differentiable, but with an additional length scale besides the hard sphere diameter. Other findings to include that 1: a longer range of the repulsive perturbation tail, or a bigger jump of the repulsive perturbation tail at an interrupted point, can stabilize the GLT more easily; 2: all of the GLTs resulting from the investigated PRPs is accompanied with a density anomaly, in contrast to the traditional GLTs due to a hard core plus an attractive tail. Finally, contrary to the previous findings in literature due to conventional 1 st-order TPT, and 2nd-order TPT based on a macroscopic compressibility approximation (MCA), the present investigation does not discover for a square shoulder (SS) potential in any periodic phase behavior of critical temperature as a function of the repulsive step radius and high density liquid-low density liquid transition (HDL-LDL). A convergence analysis of the 3rd-order TPT indicates that the previously found SS potential phenomenology (Phys. Rev. E. 2003, 67, 010201(R); Phys. Rev. E. 2006, 74, 041201), should be an artifact originating from the insufficiency of the employed 1st-order TPT and 2nd-order MCA-TPT. The counter examples are found to a liquid-liquid transition hypothesis (Nature, 1992, 360, 324) of the density anomaly.
\end{abstract}

\section{INTRODUCTION}

It is well known that a hard sphere system experiences, only a phase transition which corresponds to an order of centers of gravity of particles and may be called as an orderdisorder transition or crystallization [1,2]. When an extra attractive perturbation (called as attractive tail) is added to the hard sphere particles, the resultant system experiences an instability in a certain range of density and temperature, and generally a 1 st-order phase transition with no symmetry change, which may be called as gas-liquid transition (GLT), appears [3]. When a repulsive interaction tail, such as a repulsive Yukawa tail, is added to the hard sphere particles, the resultant system will not hold any GLT in the fluid phase [3].

When the repulsive perturbation has a finite length, for example, a square shoulder (SS) repulsion is added to the hard sphere particles, the resultant system denoted by Eq. (1), will experience a high density liquid (HDL)-low density liquid (LDL) transition as reported in Ref. [4].

$$
\begin{aligned}
u(r)=\propto & r / \sigma<1 \\
\varepsilon & \lambda>r / \sigma \geq 1 . \\
0 & \lambda \leq r / \sigma
\end{aligned}
$$

A more detailed investigation on the system with underlying potential (1) is carried out in Ref. [5] with help of a 1st-order thermodynamic perturbation theory (TPT). It is found that the appearance of the HDL-LDL transition,

\footnotetext{
*Address correspondence to this author at the School of Physics Science and Technology, Central South University, Changsha, Hunan, China, 410083; E-mail: chixiayzsq@yahoo.com
}

reveals some kind of periodic behavior of the critical temperature as a function of the repulsive step radius $\lambda$, the periodic behavior is considered in Ref. [5] to be obviously correlated with the behavior of the hard sphere radial distribution function (rdf). It is recorded [5] that 'for small values of $\lambda$, there is no phase transition, the HDL-LDL transition appears in the vicinity of $\lambda \approx 1.5$ and disappears again for $\lambda \approx 2$. On further increase of $\lambda$, the isotherms reveal some tendencies to the formation of a van der Waals loops'.

The HDL-LDL transition reported in Refs. 4-5 for system with the underlying SS potential (1) happens in very high density region, the critical reduced density is about 1 (here reduced density is defined as $\rho^{*}=\rho \sigma^{3}$ with $\rho=N / V$ a number density, where $\mathrm{N}$ is the particle number and $\mathrm{V}$ is the volume are occupied by the system, respectively, while $\sigma$ shows the hard sphere core diameter). In a recent investigation [6], a 2nd-order TPT based on a macroscopic compressibility approximation (MCA) (2nd-order MCA-TPT), which is also employed in Ref. [4], is used to investigate the HDL-LDL transition system of the spherical particles interacting via a core-softened attractive (CSA) potential, combining a repulsive square shoulder and an attractive square well [7]. It is found that the predicted critical densities of the HDL-LDL transition are far larger than the corresponding simulation results [7], the predicted critical temperatures also deviate largely, from the simulation values. Therefore, the conventional 2nd-order MCA-TPT may not be reliable sufficiently, for these discontinuous soft repulsive potentials, the conclusions drawn from the 2nd-order MCA-TPT or 1storder TPT calculation may not be accurate quantitatively, or 
even problematic qualitatively $[4,5]$. There exists a necessity to employ more accurate theoretical approach to investigate these purely repulsive, discontinuous potentials. In Ref. [6], a recently proposed 3rd-order TPT [8] by the present author, is employed to predict the critical parameters of the CSA potential. It is found that the critical parameters predicted from the 3rd-order TPT are in a very satisfactory agreement with the corresponding simulation results even for cases, where the predictions from the 2nd-order MCA-TPT are very poor or even incorrect qualitatively. Therefore, the 3rdorder TPT is well qualified for these discontinuous potentials with a soft repulsion.

The organization of the paper is as follows. In Sec.II, the 3 rd-order TPT is recapitulated firstly, then a convergence analysis of the 3rd-order TPT is given. The 3rd-order TPT is then in the Sec.II used to investigate the phase transition properties in the fluid phase of several model fluids interacting, through various PRPs, also a detailed analysis is given to the theoretically calculated results. Finally, in Sec.III the paper is ended by summarizing theoretical results reported presently, about the phase transitions in the fluid phase of the PRP systems.

\section{THE 3RD-ORDER TPT AND PHASE TRANSI- TIONS OF SYSTEMS WITH UNDERLYING PRPS}

The 3rd-order TPT [8] is given by:

$$
\begin{gathered}
F_{e x}=F_{e x-r e f}+F_{e x-T a i l}, \text { and } F_{e x-\text { Tail }}=\sum_{n=1}^{3} F_{e x-n} \\
F_{e x-n}=\left.\frac{1}{n !} N 2 \pi \rho \int d r r^{2} u_{p e r}(r) \frac{\partial^{(n-1)} g(r, \lambda, \rho)}{\partial \lambda^{(n-1)}}\right|_{\lambda=0} .
\end{gathered}
$$

Here, $F_{e x-r e f}$ is an excess Helmholtz free energy of a reference hard sphere fluid with a potential $u_{r e f}, u_{p e r}(r)$ is perturbation part of the whole potential $u(r)$ given by:

$u(r)=u_{r e f}(r)+u_{p e r}(r)$.

In the present form of the 3rd-order TPT [8], $u_{r e f}(r)$ is the hard sphere potential given by:

$$
\begin{aligned}
u_{r e f}(r)=\propto, & r<\sigma \\
0, & r>\sigma
\end{aligned}
$$

$g(r, \lambda, \rho)$ is the $\operatorname{rdf}$ of bulk fluid with pair potential $F_{e x}(\lambda)=-k T \ln \left(\int d \mathbf{r}^{N} e^{-\beta \sum_{i<j} u\left(r_{i j} ; \lambda\right)} / V^{N}\right)$ given by:

$u(r ; \lambda)=u_{r e f}(r)+\lambda u_{p e r}(r)$.

$\left.\frac{\partial^{(n-1)} g(r, \lambda, \rho)}{\partial \lambda^{(n-1)}}\right|_{\lambda=0}$

is the (n-1)th derivative evaluated at $\lambda=0$ of $g(r, \lambda, \rho)$ with respect to $\lambda$, $\left.\frac{\partial^{0} g(r, \lambda, \rho)}{\partial \lambda^{0}}\right|_{\lambda=0}=g(r, 0, \rho)$

is the rdf of the hard sphere fluid of density $\rho$ and diameter $\sigma$. As done in Ref. [8],

$\left.\frac{\partial^{(n-1)} g(r, \lambda, \rho)}{\partial \lambda^{(n-1)}}\right|_{\lambda=0}$

for $n=1,2,3$ in the 3rd-order TPT is calculated by an Ornstein-Zernike (OZ) integral equation theory (IET). The $F_{e x-r e f}$ is calculated by a Cananhan-Startling equation of state [9]. The readers can consult Ref. [8] for calculational details. The 1st-order and 2nd-order TPTs result when the $F_{e x-2}, F_{e x-3}$ and $F_{e x-3}$ are respectively discarded away.

In the 2nd-order MCA-TPT, the excess Helmholtz free energy $F_{e x}$ is given by

$$
\begin{aligned}
F_{e x}= & F_{e x-r e f}+N 2 \pi \rho \int d r r^{2} u_{p e r}(r) g(r, 0, \rho)- \\
& N \beta^{2} \pi \rho \int d r r^{2} u_{p e r}{ }^{2}(r) g(r, 0, \rho) \frac{1}{\beta}\left(\frac{\partial \rho}{\partial P}\right)_{r e f},
\end{aligned}
$$

here, $\beta=\frac{1}{k T}$ with $k$ Boltzmann constant and $T$ absolute temperature. The compressibility $\frac{1}{\beta}\left(\frac{\partial \rho}{\partial P}\right)_{\text {ref }}$, and $\operatorname{rdf}$ $g(r, 0, \rho)$ of the reference hard sphere fluid can be calculated by various routes. In Refs 4 and 5 they are obtained by a Percus-Yevick (PY) approximation for the OZ IET.

In Fig. (1), we present the hard sphere rdf,

$\left.\frac{\partial g(r, \lambda, \rho)}{\partial \lambda}\right|_{\lambda=0}$,

and $\left.\frac{\partial^{2} g(r, \lambda, \rho)}{\partial \lambda^{2}}\right|_{\lambda=0}$

for the SS potential denoted by Eq. (1), whereas the corresponding results for a square well (SW) potential denoted by Eq. (8), is presented in Fig. (2),

$$
\begin{aligned}
& u(r)=\infty \quad r / \sigma<1 \\
& -\varepsilon \quad \lambda>r / \sigma \geq 1 \\
& 0 \quad \lambda \leq r / \sigma
\end{aligned}
$$

By observing Fig. (1) and (2), one can find that the absolute values of

$$
\left.\frac{\partial g(r, \lambda, \rho)}{\partial \lambda}\right|_{\lambda=0} \text { and }\left.\frac{\partial^{2} g(r, \lambda, \rho)}{\partial \lambda^{2}}\right|_{\lambda=0}
$$

are generally smaller than that of the hard sphere rdf at the same interparticle separation for case of the SW fluid, therefore the 2nd-order term and 3rd-order term ignored in the 1storder TPT should be smaller quantities compared with the 1storder term, the ignorance of the 2nd-order term and 3rd-order 
term may not be an ackward approximation, the resultant prediction is at least qualitatively or even quantitatively correct. For the PRP, on the contrary, the absolute values of

$\left.\frac{\partial g(r, \lambda, \rho)}{\partial \lambda}\right|_{\lambda=0}$ and $\left.\frac{\partial^{2} g(r, \lambda, \rho)}{\partial \lambda^{2}}\right|_{\lambda=0}$

are even larger than that of the hard sphere rdf itself, therefore the 2nd-order term and 3rd-order term ignored in the 1st-order TPT are not smaller quantities compared with the 1 st-order term, they contribute significantly to the total $F_{e x}$. To illustrate the above analysis quantitatively, we present the calculated critical parameters for the HDL-LDL transition of the CSA potential [7] in Table 1, from which several conclusions can be drawn. The 2nd-order MCA-TPT only improves upon the 1st-order TPT a little, this is in agreement with the observation in Ref. [8] that the 2nd-order term of the perturbation expansion is severely underestimated by the MCA, when the value of $\rho^{*}$ is high. The present 2 nd-order TPT improves largely upon the 1st-order TPT and the 2nd-order MCA-TPT, this indicates that the perturbation expansion is convergent upto at least 2nd-order and the 2nd-order term can be tackled more accurately, by the present numerical method than by the MCA. The fact that the 3rd-order TPT again largely improves upon the 2nd-order TPT indicates that the perturbation expansion is also convergent at 3rdorder and the retaining of the 3rd-order term is required for an improvement of accuracy of the TPT. We present the numerical values of each expansion term of the TPT in Table 2, from which one finds that the magnitude of the 2nd-order term is even larger than that of the 1st-order term, the MCA underestimate severely, the magnitude of the 2nd-order term, the magnitude of the 3rd-order term is smaller than those of the 1st-order and 2nd-order term, but its incorporation into the perturbation expansion can significantly improve the accuracy of the resultant TPT. Therefore the appropriate order of the TPT should be the third order, and the 3rd-order TPT satisfactorily accounts for the thermodynamic properties of the CSA potential. From Table 1 shows that the 1storder TPT and 2nd-order MCA-TPT largely overestimates the critical density of the HDL-LDL transition of the CSA potential. Therefore, it is reasonable to suspect that the unusual high critical density of the SS potential predicted by the 1st-order TPT and 2nd-order MCA-TPT $[4,5]$ is an artifact originating from the insufficiency of the 1st-order TPT and 2nd-order MCA-TPT, and there is a necessity to employ the present 3rd-order TPT for investigation of the SS potential. In fact, the present author finds in Ref. [6] that the 2ndorder MCA-TPT fails to predict the appearance of the HDLLDL transition of the CSA potential for several parameters combinations, even if the simulation establishes the existence of the HDL-LDL transition.

Firstly, we calculate for the SS potential denoted by (1), the chosen values of the repulsive step radius $\lambda$ is equal to $1.25,1.5,2$ and 2.5 respectively. The reduced pressure $\left(P \sigma^{3} \beta\right)$-reduced density $\rho^{*}$ isotherms for each case are presented in Fig. (3a-d). Unlike the conclusion obtained in Ref. [5], we have not found any periodic behavior of the transi- tion critical temperature as a function of the $\lambda$, the 1 st-order phase transition happens to each case, the reduced critical temperature $T_{c}^{*}=k T_{c} / \varepsilon \quad\left(T_{c}\right.$ is absolute critical temperature) increases monotonously, as the $\lambda$ increases. To show that the monotonous behavior applies for each section of the range of the $\lambda$, we present in Fig. (4) the $T_{c}^{*}-\lambda$ function with the $\lambda$ situated around 1.5 and 2 respectively. From Fig. (4) one can see that the $T_{c}^{*}$ still monotonously increases as the $\lambda$ increases, this observation turns out contrary to the conclusion drawn in Ref. [4], in which the 2nd-order MCATPT predict that the $T_{c}^{*}$ decreases, when the $\lambda$ increases from $\lambda=1.4$ to $\lambda=1.6$.
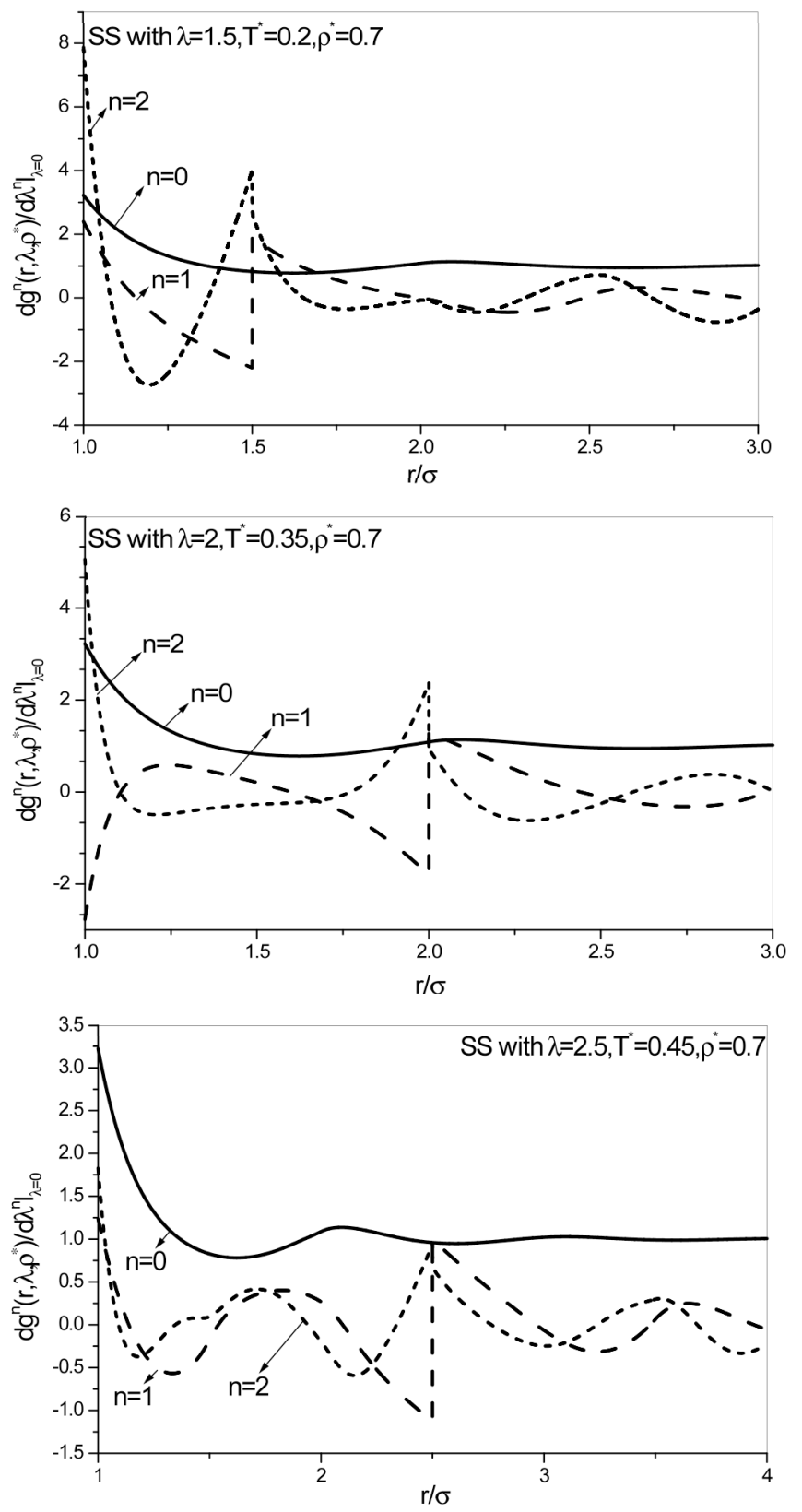

Fig. (1). The $\left.\frac{\partial^{(n)} g(r, \lambda, \rho)}{\partial \lambda^{(n)}}\right|_{\lambda=0}$ with $\lambda=0,1,2$ for the SS fluid at three state points. 
Table 1. Temperature $T_{C_{2}}$, Pressure $P_{C_{2}}$, and Density $\rho_{C_{2}}$ for the Critical Point $C_{2}$ of the CSA Potential in Ref. [7], Respectively Computed by MD Simulation Method [7] (1st Line), 3rd-Order TPT (2nd Line), 2nd-Order MCA-TPT (3rd Line), 1st-Order TPT (4th Line), and 2nd-Order TPT (5th Line). The Potential Parameters of Each Case Can Be Found in Ref. [7], the Symbols $a$ and $U_{A}$ have the Same Meanings as in Ref. [7]

\begin{tabular}{|c|c|c|c|}
\hline \multirow{3}{*}{ Set } & $\boldsymbol{k} \boldsymbol{T}_{\boldsymbol{C}_{2}} / \boldsymbol{U}_{\boldsymbol{A}}$ & $\boldsymbol{a}^{3} \boldsymbol{P}_{\boldsymbol{C}_{2}} / \boldsymbol{U}_{\boldsymbol{A}}$ & $\boldsymbol{a}^{3} \boldsymbol{\rho}_{\boldsymbol{C}_{2}}$ \\
\hline \hline \multirow{3}{*}{ (ix) } & $0.53 \pm 0.03$ & $1.05 \pm 0.03$ & $0.39 \pm 0.05$ \\
\cline { 2 - 4 } & 0.5456 & 0.2843 & 0.4124 \\
\cline { 2 - 4 } & 1.5943 & 12.8694 & 0.9323 \\
\cline { 2 - 4 } & 1.6023 & 12.2189 & 0.9251 \\
\cline { 2 - 4 } & 0.1105 & -2.6066 & 0.4912 \\
\hline \multirow{3}{*}{ (x) } & $0.57 \pm 0.01$ & $0.58 \pm 0.01$ & $0.35 \pm 0.02$ \\
\cline { 2 - 4 } & 0.6632 & 0.3252 & 0.4166 \\
\cline { 2 - 4 } & 1.7333 & 9.8503 & 0.8912 \\
\cline { 2 - 4 } & 1.7545 & 9.3385 & 0.8945 \\
\cline { 2 - 4 } & 0.2087 & -0.9404 & 0.4821 \\
\hline
\end{tabular}

Table 2. The n-Order Term of the Perturbation Expansion for the CSA Potential in Ref. [7], the Potential Parameters of Set ix and $x$ Can Be Found in Ref. [7], the Symbols $a$ and $U_{A}$ have the Same Meanings as in Ref. [7].

\begin{tabular}{|c|c|c|}
\hline & Set (ix) & Set $(x)$ \\
\hline & $k T / U_{A}=0.7 \quad a^{3} \rho=0.7$ & $k T / U_{A}=0.7 \quad a^{3} \rho=0.7$ \\
\hline 1 st-order term & 5.345143 & 2.378280 \\
\hline 2 nd-order term & -8.095391 & -8.125361 \\
\hline 2nd-order term ${ }^{\mathrm{MCA}}$ & -1.482945 & -1.603198 \\
\hline 3rd-order term & -0.522470 & -0.1086290 \\
\hline
\end{tabular}

The periodic behavior is correlated in Ref. [5] with the behavior of the hard sphere $\mathrm{rdf}$ as follows. Since $r \approx 1.5 \sigma$, $r \approx 2 \sigma, r \approx 2.5 \sigma$ approximately correspond to the first minimum, second maximum, and second minimum of the hard sphere rdf, from Eq. (7) one can see that for $\lambda \approx 1.5$ the contribution to the system free energy comes from the first coordination sphere, which changes most drastically under the fluid-fluid phase transition. A similar situation also takes place for $\lambda \approx 2.5$, where the contribution corresponds to the first and second coordination shells. For $\lambda \approx 2$ the contribution is more smooth and hence, the transition will not result. In the present 3rd-order TPT, besides the first order term associated with the hard sphere rdf itself, there are two additional second order and third order terms respectively associated with the first order and second order derivative of the rdf, i.e. $\left.\frac{\partial g(r, \lambda, \rho)}{\partial \lambda}\right|_{\lambda=0}$ and $\left.\frac{\partial^{2} g(r, \lambda, \rho)}{\partial \lambda^{2}}\right|_{\lambda=0}$.

As shown in Fig. (1) for the SS potential, the $\left.\frac{\partial g(r, \lambda, \rho)}{\partial \lambda}\right|_{\lambda=0}$ and $\left.\frac{\partial^{2} g(r, \lambda, \rho)}{\partial \lambda^{2}}\right|_{\lambda=0}$

are structured largely differently from the hard sphere rdf, the formers can even be valued negatively. Obviously, the expected periodicity of the theoretical results due to the first order term is largely destroyed due to inclusion of the $\left.\frac{\partial g(r, \lambda, \rho)}{\partial \lambda}\right|_{\lambda=0}$ and $\left.\frac{\partial^{2} g(r, \lambda, \rho)}{\partial \lambda^{2}}\right|_{\lambda=0}$.
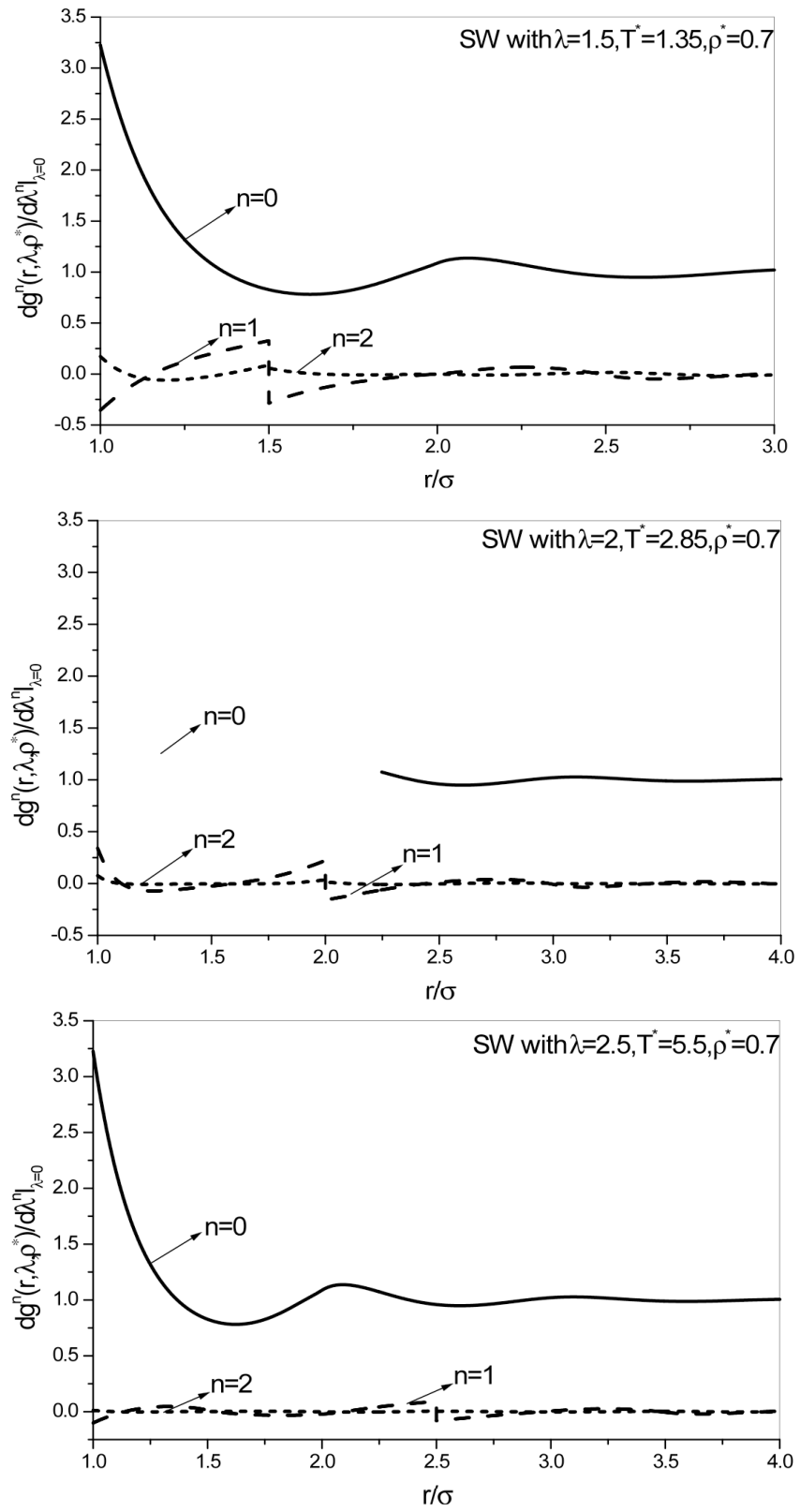

Fig. (2). The $\left.\frac{\partial^{(n)} g(r, \lambda, \rho)}{\partial \lambda^{(n)}}\right|_{\lambda=0}$ with $\lambda=0,1,2$ for the SW fluid at three state points. 

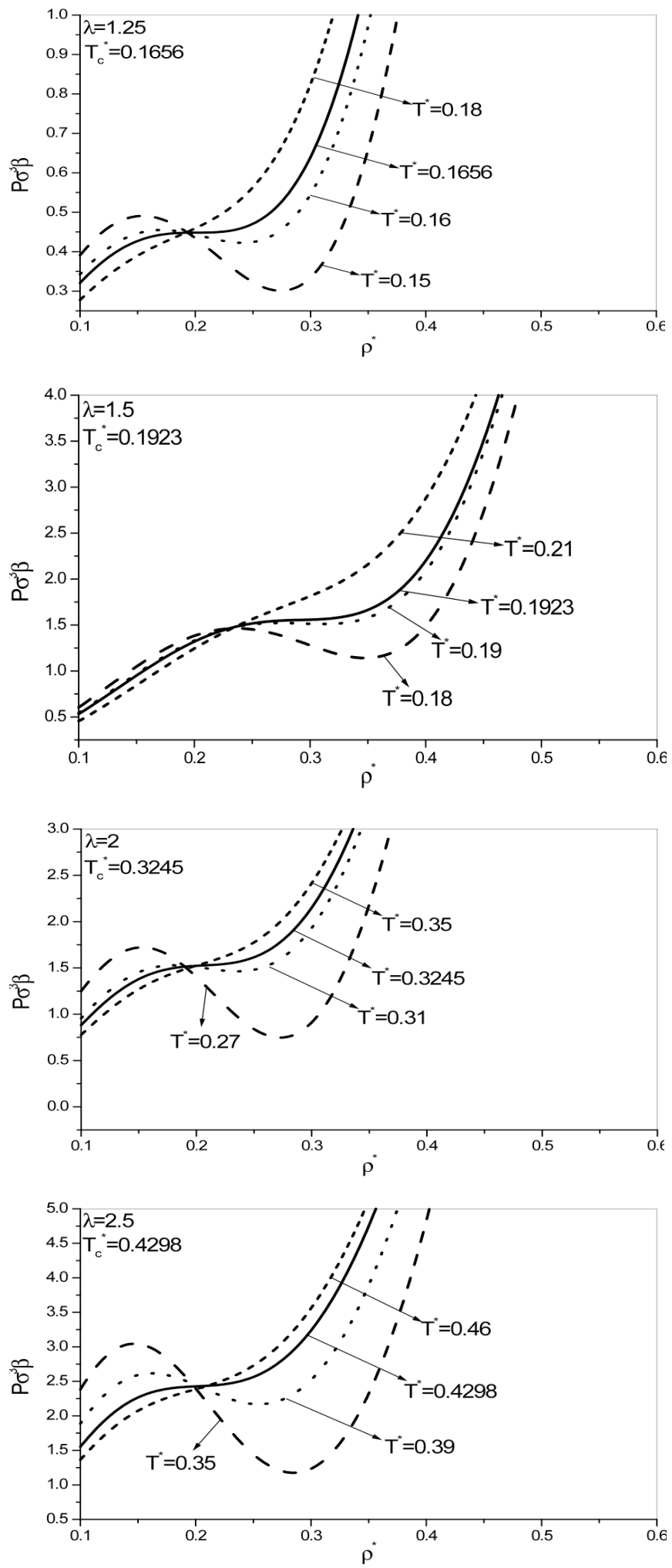

Fig. (3). The families of the pressure-density isotherms for the SS potential for different values of $\lambda$.

Therefore, the resultant $F_{e x}$ in the 3rd-order TPT will not be of periodicity. It should be noted that although the 2 nd-order MCA-TPT incorporates the 2nd-order term into itself, the 2nd-order term is associated with the hard sphere rdf, not with the derivative of the rdf as done in the 3rd-order TPT. Therefore, as pointed out in Ref. [5] the phase transition pre- dicted [4] by the 2nd-order MCA-TPT for the SS potential is still periodic as in the 1 st-order TPT.

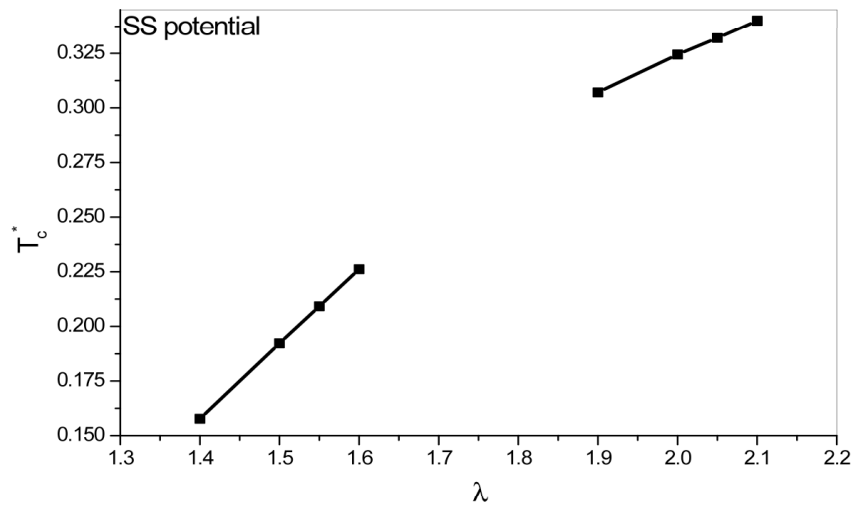

Fig. (4). The critical temperature $T_{c}^{*}$ as a function of the step radius $\lambda$ for the SS potential system.

The fluid phase coexistence curve for the SS potential is presented in Fig. (5) for potential parameter $\lambda=2$ and $\lambda=5$ respectively. As the critical reduced density $\rho_{c}^{*}$ is low, what is shown in Fig. (5) is obviously a GLT curve, in contradiction to the LDL-HDL transition reported in Refs. 4, 5 with the help of the 2nd-order MCA-TPT and the 1st-order TPT respectively.
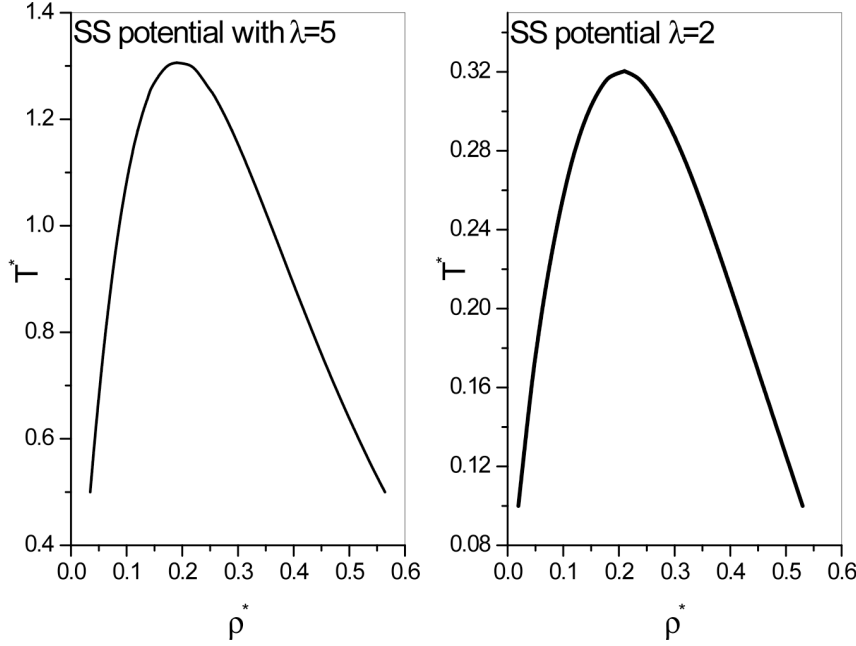

Fig. (5). The 1st-order phase transition in fluid state of the SS potential system with $\lambda=2$ and $\lambda=5$.

The GLT in the purely repulsive fluids is a challenge to the traditional van der Waals picture of fluid phase transition, which attributes the GLT to an attractive tail added to a steeply repulsive hard core. To confirm whether the GLT of the purely repulsive SS potential is due to an artifact of discontinuity of the perturbation potential or not, we define a new potential denoted by Eq. (9) whose special situation is exactly the SS potential,

$$
\begin{array}{cc}
u(r)=\propto & r / \sigma<1 \\
\varepsilon\left[1+\frac{(1-\alpha)(r-1)}{(1-\lambda)}\right] & \lambda>r / \sigma \geq 1 . \\
0 & \lambda \leq r / \sigma
\end{array}
$$


Here, $\alpha \geq 0$ corresponds to the presently investigated PRP. When $\alpha=0$, the perturbation potential becomes continuous; When $\alpha=1$, the potential reduces to the SS potential (1). Value of the $\alpha$ determines the jump of the potential at the interrupted point $r=\lambda \sigma$, a larger $\alpha$ corresponds to a larger jump at the interrupted point. In Fig. (6), the potential is shown for the parameters $\lambda=2$ and $\alpha=0,0.5,1,1.5,2$ respectively. The $T_{c}^{*}$ as a function of the $\alpha$ for constant $\lambda=2$ is presented in Fig. (7), which clearly reveals that the $T_{c}^{*}$ decreases, when the $\alpha$ decreases. But even if the $\alpha$ becomes equal to zero, i.e. the perturbation potential becomes continuous, the GLT still appears. Therefore, one concludes that the discontinuity of the perturbation potential is not a necessary condition for the presence of the GLT in the PRP, the GLT can happen to have a hard sphere plus purely repulsive and continuous perturbation potential, whose perturbation part includes an indifferentiable point. The $T_{c}^{*}$ as a function of the $\lambda$ for constant $\alpha=0$ is presented in Fig. (8), which shows that the $T_{c}^{*}$ is an increasing function of $\lambda$. As in the case of $\alpha=1$, the $\alpha=0$ case also does not display any periodic behavior of the transition critical temperature as a function of the $\lambda$.

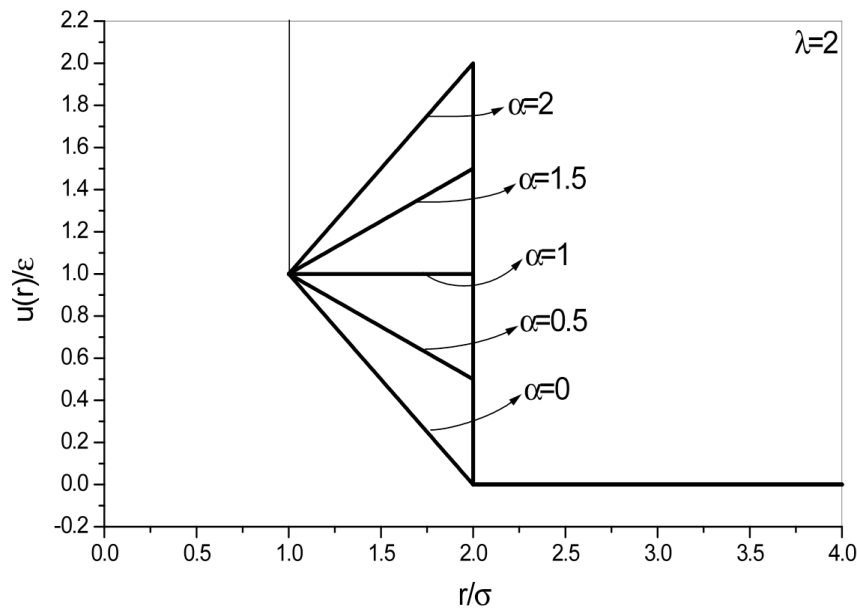

Fig. (6). The potential Eq. (9) for different $\alpha$ but fixed $\lambda=2$.

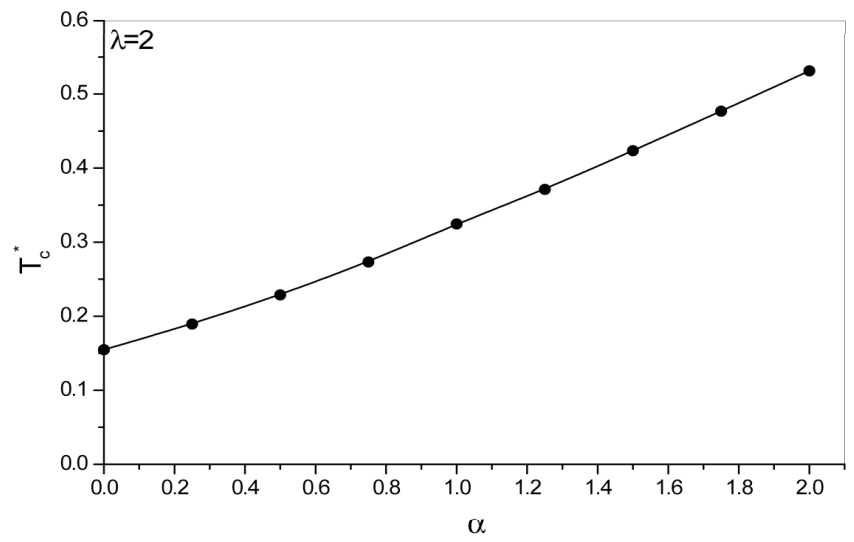

Fig. (7). The critical temperature $T_{c}^{*}$ as a function of the potential parameter $\alpha$ for the potential Eq. (9) with $\lambda=2$.

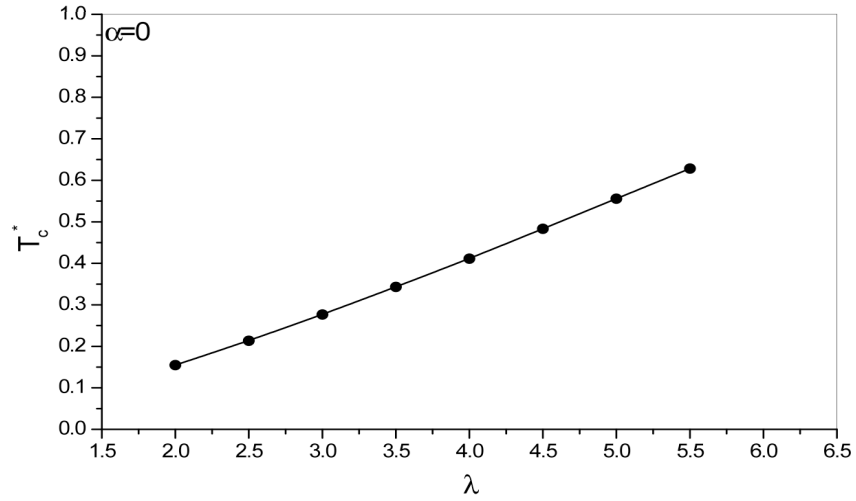

Fig. (8). The critical temperature $T_{c}{ }^{*}$ as a function of the potential parameter $\lambda$ for the potential Eq. (9) with $\alpha=0$.

To indicate that the GLT generally happens to the PRP, we employ a truncated hard core repulsive Yukawa (HCRY) potential as the additional sample potential,

$$
\begin{array}{cc}
u(r)=\propto & r / \sigma<1 \\
\varepsilon \sigma \exp \left[-\kappa^{*}(r-\sigma) / \sigma\right] / r & \lambda>r / \sigma \geq 1, \\
0 & \lambda \leq r / \sigma
\end{array}
$$

when $\lambda=\propto$, the truncated HCRY potential reduces to the HCRY potential itself. It is well-known that the fluid phase of the HCRY potential is stable, and does not display any phase transition. However, when the HCRY potential is truncated at some finite $r=\lambda \sigma$, new situation appears. The pressuredensity isotherms for two cases of the truncated HCRY potential are presented in Figs. $(\mathbf{9 a}, \mathbf{b})$ and $(\mathbf{1 0})$, which show that the system with the underlying truncated HCRY potential experiences are surely instable in certain fluid phase domain.
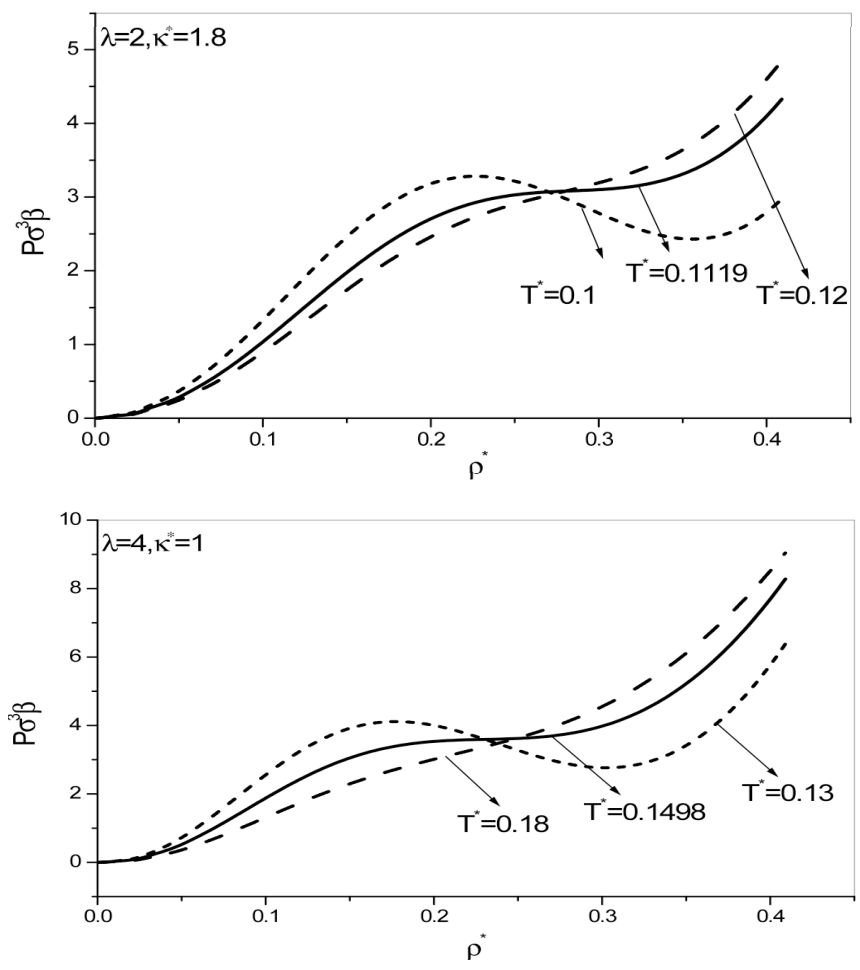

Fig. (9). The families of the pressure-density isotherms in fluid state of the truncated HCRY potential (10). 
To further demonstrate, the generality of the GLT in fluid phase of the PRPs, we construct a new sample potential given by

$$
\begin{aligned}
u(r)= & & r / \sigma< \\
\varepsilon\left[1+\frac{(1-\alpha)(r-1)}{(1-\lambda)}\right] & & \lambda>r / \sigma \\
& \alpha \varepsilon \lambda \exp \left[-\kappa^{*}(r-\lambda) / \sigma\right] / r & \lambda \leq r / \sigma
\end{aligned}
$$

this potential consists of a linear ramp and a RY tail besides the hard sphere repulsion. Obviously, the potential function is indifferentiable at $r / \sigma=\lambda$. The pressure-density isotherms for one case of the potential (11) are presented in Figs. (10) and (11). By comparing the Figs. (10) and (11) with the Figs. (7) and (8) one can see that when a continuous RY tail is added to the ramp repulsion, the instability appear more easily, the $T_{c}{ }^{*}$ becomes higher. This observation is in agreement with the Figs. (4-3,5-4,9-8) which indicate that the $T_{c}^{*}$ increases as the potential range increases.

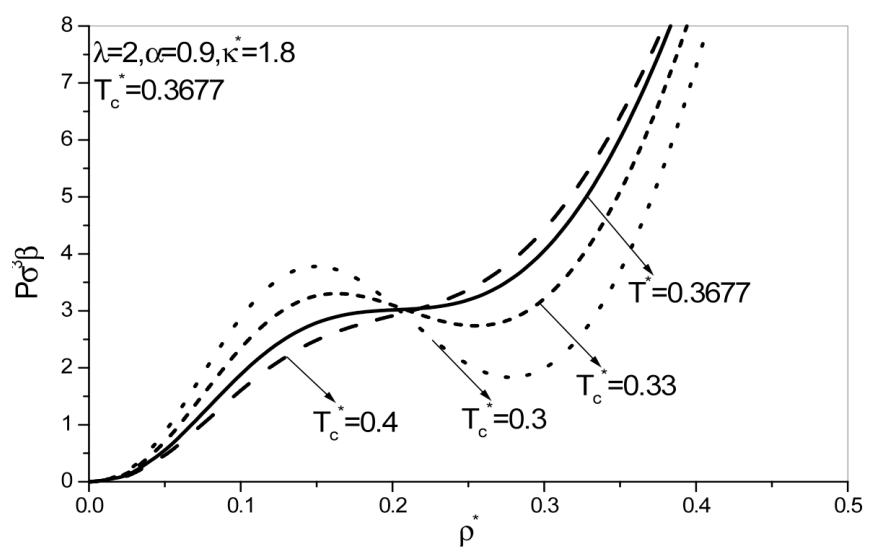

Fig. (10). The families of the pressure-density isotherms for the potential (11).

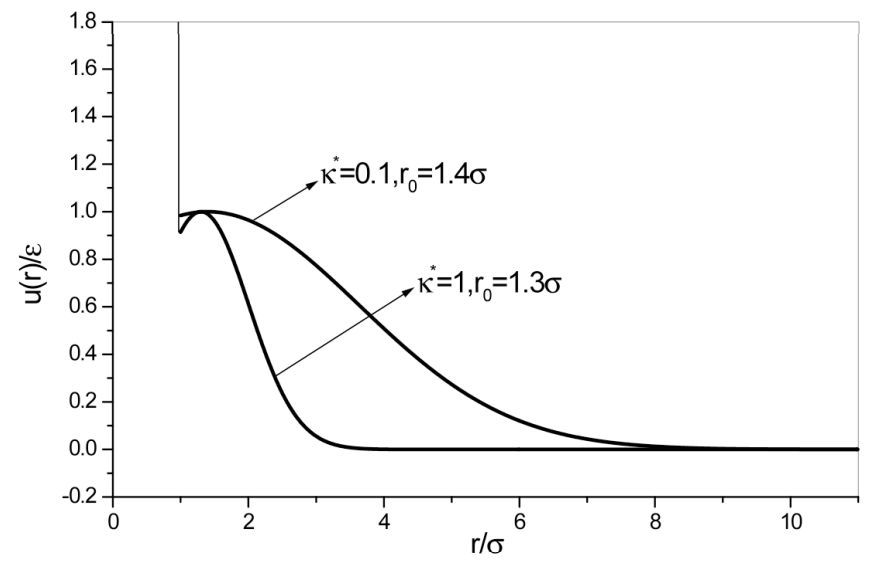

Fig. (11). The potential (12) for two sets of potential parameters as shown in the figure.

To indicate that the indifferentiability is also not necessary condition for appearance of the fluid phase, instability in the purely repulsive systems, we will investigate a truncated and shifted Gauss-like potential added to the hard sphere potential given by;

$$
\begin{array}{ll}
u(r)=\propto & r / \sigma<1 \\
\varepsilon \exp \left[-\kappa^{*}\left(\left(r-r_{0}\right) / \sigma\right)^{2}\right] & r / \sigma \geq 1
\end{array} .
$$

In Fig. (11), the potential Eq. (12) is shown for two sets of parameter value: $\kappa^{*}=1, \quad r_{0}=1.3 \sigma$ and $\kappa^{*}=0.1$, $r_{0}=1.4 \sigma$ respectively. Potential (12) is continuous and differentiable, but the potential slope changes sign at some particle separation. The pressure-density isotherms for two cases of the potential (12) are presented in Fig. (12), which clearly shows that the continuous, differentiable, purely repulsive perturbation combined with the hard sphere repul-
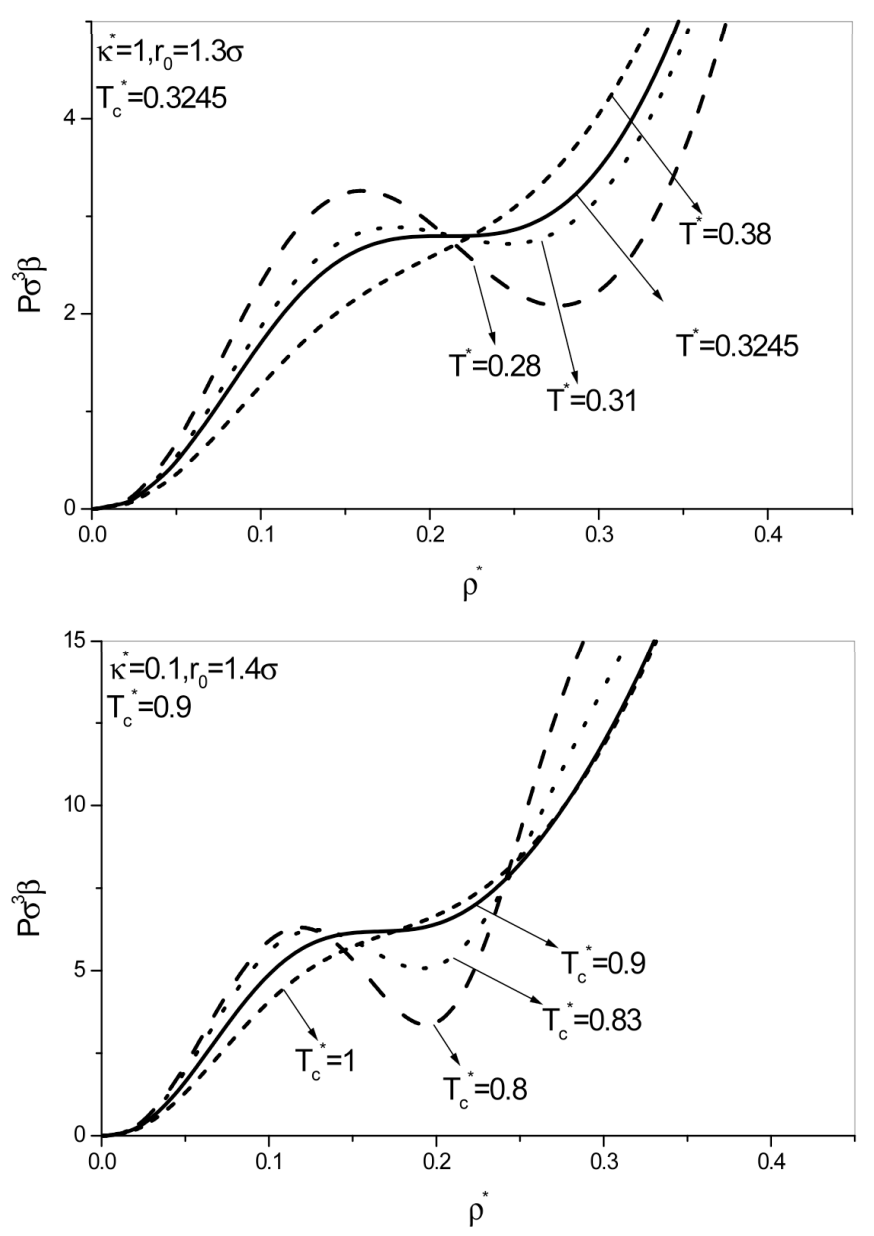

Fig. (12). The families of the pressure-density isotherms for the potential (12).

sion also experiences instability, and displays the GLT whose coexistence curve is not presented for simplicity. The only difference between the potential (12) and the complete HCRY potential free of fluid phase instability, is that the RY perturbation is a monotonous function of the particles separation $r$, but the perturbation part of the potential (12) is a non-monotonous function of the $r$, the perturbation part of the potential (12) has a extreme value at $r=r_{0}$, whose existence induces an additional length scale. It is exactly the combination of the additional length scale and the original length scale, i.e. the hard sphere diameter $\sigma$, that induces the fluid phase instability in the hard sphere plus purely re- 
pulsive, continuous and differentiable perturbation tail. Here the second length scale or two scale potentials normally refers to potentials that have two relevant ranges of the repulsive interaction. For instance, the standard ramp potential has a first length scale, which is the hard core, and a second length that is the range of the repulsive ramp. In the case of the one scale ramp potential the hard core is missing and one has a repulsive ramp down to zero separation. Existing literatures [10-14] indicate that the additional length scale situated in the soft repulsion region of the potential, when combined with an attractive interaction at larger separation, can induce a HDL-LDL transition, the present investigation reveals that the additional length scale, unaccompanied by addition of the attractive tail, singly can induce the GLT. Therefore, one can conclude that the additional length scale may be a more essential property for appearance of fluid phase instability in systems with hard sphere plus purely repulsive perturbation. In fact, all of the above investigated sample potentials, which display the instability in the fluid phase, are all endowed with an additional length scale besides the hard sphere diameter, one can see this point clearly from Figs. (6) and (11) and Eqs.(1,9-12).

Density anomaly is a common phenomena, occurring in many molecular liquids such as water, silica and carbon [1517]. Usually it is thought that the density anomaly is due to a HDL-LDL transition [18]. There exists a variety of indirect experimental and theoretical data favoring the HDL-LDL transition hypothesis [18] of the density anomaly. Figs. $(3,9,10,12)$ show that the pressure isotherm cross each other, which implies a density anomaly since $\left(\partial \rho^{*} / \partial T^{*}\right)_{P}=0$ In Figs. $(\mathbf{1 3 a}, \mathbf{b})$ the density as a function of temperature for different pressures is presented for potential $(11,12)$, one clearly detects a density maximum and minimum. Considering that all of these systems only experiences the GLT, the present investigation, therefore supplies counter examples to the HDL-LDL transition hypothesis [18] of the density anomaly.

To explain why the GLT is never previously observed in computer simulation for the PRP, we present in Fig. (14) the phase diagram of the potential (12). To reduce the computational time for calculation of the solid phase free energy, the potential (12) is truncated and shifted at $r=8 \sigma$, facecentered cubic (fcc) solid phase free energy is calculated by a method detailed in Ref. [19]. An unusual finding is that the GLT is always metastable with respect to the order-disorder transition. Thus, we explain why the GLT has not been observed in computer simulation, possibly because the metastable lifetime of these gas-liquid co-existence phases is too small to be resolved by computer simulation. Although, the GLT is metastable, its existence is not senseless. The recent experiments, numerical calculations, and theoretical considerations [20-22] have shown that the presence of a metastable GLT critical point drastically changes the pathway for the formation of a crystal nucleus in solutions of globular proteins. Close to the critical point, the free energy barrier for crystal nucleation is strongly reduced, Hence, the crystal nucleation rate increases by many orders of magnitude.
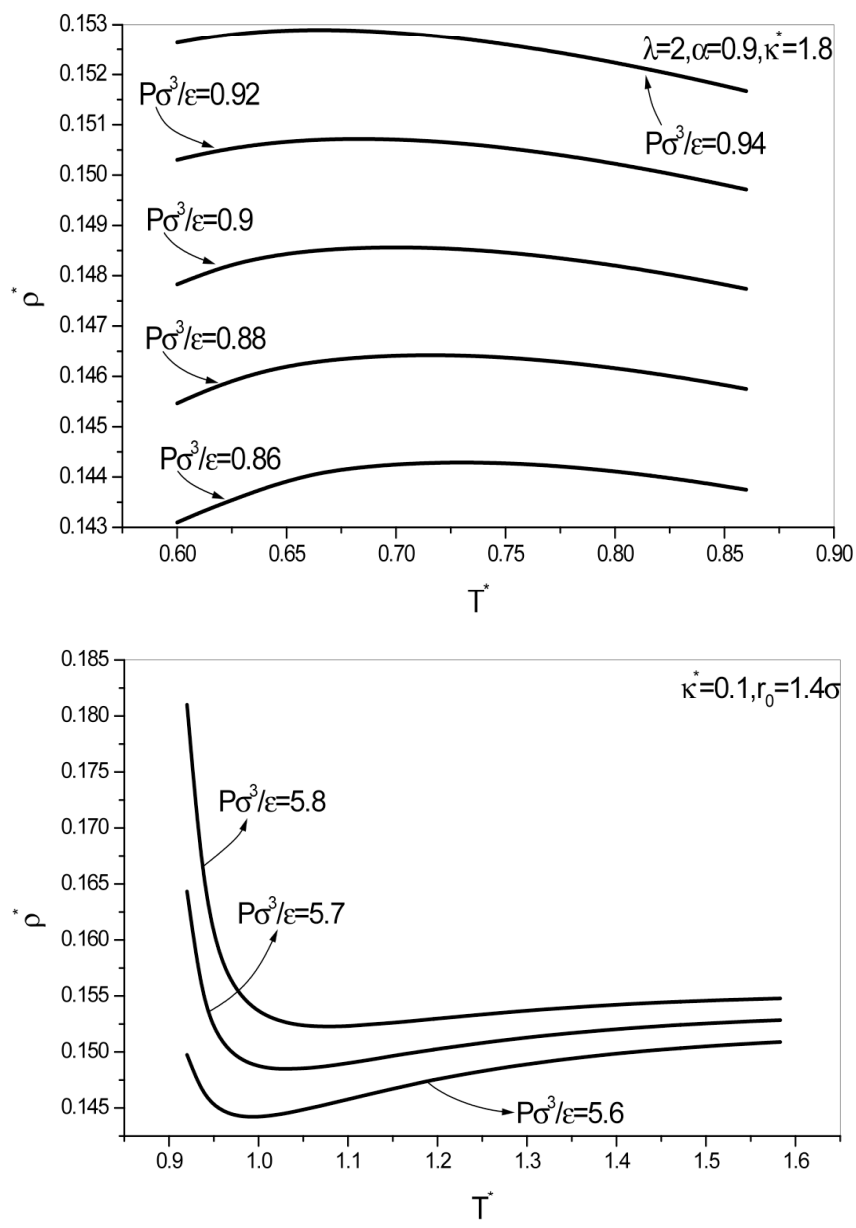

Fig. (13). The density $\rho^{*}$ as a function of temperature $T^{*}$. Subfigure (a) is for potential (11), Subfigure (b) is for potential (12). The potential parameters are shown in the figures.

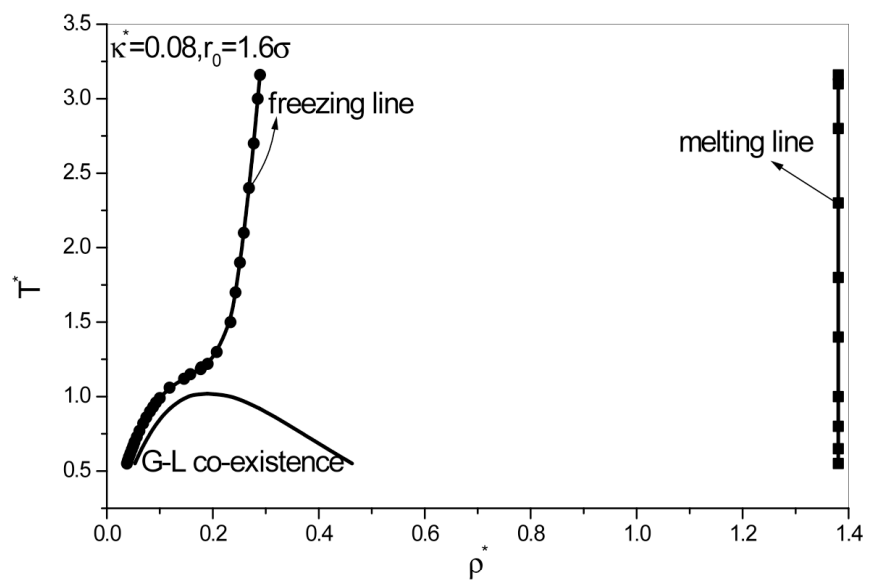

Fig. (14). Phase diagram of the potential (12) truncated and shifted at $r=8 \sigma$. The potential parameters are shown in the figure.

\section{SUMMARIES}

With the help of the accurate 3rd-order TPT, we investigate in detail, the 1st-order phase transition in fluid phase of systems of particles, interacting through the PRPs. It is found that the 1st-order GLT generally exists in the PRPs, whose perturbation part holds on an additional length scale besides the hard sphere diameter, in contradiction to the traditional van der Waals picture of the fluid phase transition, which declares 
that the GLT is necessarily induced by appending an attractive perturbation to the hard sphere potential. Concretely speaking, we find that the 1st-order GLT occurs generally in system of the PRPs, whose perturbation parts include a discontinuous point, or an indifferentiable point, or a sign change of the slope. In molecular liquids and complex fluids [23, 24], the underlying potentials are of a form of an effective potential usually originating from an average over the angular part of more realistic potentials or integration of free degree of small particles in the systems, the resultant effective potential is easily endowed with the additional length scale. Therefore the GLT generally occurring in these complex fluids and molecular liquids [25] does not conflict with the present conclusion.

As for the reason, why the additional length scale induces the otherwise non-existent GLT? It can be explained that it happens due to two preferred interparticle distances resulting from the two length scales. Thus, the system assumes a open structures at low pressure, and transforms to more compact structures at higher pressures, this corresponds to a collapse from the largest distance $\lambda \sigma$ or $r_{0}$ to the smallest distance $\sigma$ on applying pressure. The main difference of the GLT associated with the PRPs from that occurring in the traditional atomic fluids, whose particles interact through hard sphere repulsion plus an attractive tail, is that the former is often accompanied with the density anomaly, while the pressure isotherms never cross each other for the latter, and therefore the density anomaly never occurs for the latter.

Besides the theoretical significance of the PRPs, the investigation of the PRPs is also closely connected with the CSA potential [7]. In Ref. [7], it is shown that the HDL-LDL transition can happen unaccompanied by the density anomaly, considering that the density anomaly surely appears in the PRP fluids, therefore, one can conclude that a combinational influence from the soft repulsion at smaller interparticle distance and an attractive tail at larger interparticle distance can remove away the density anomaly and at the same time induce the HDL-LDL transition. The combinational influence from the soft repulsion and attractive tail makes the investigation of the CSA potential [7] a very interesting problem [26-28], but far away from solution. It may be a feasible route to uncover the mechanism for the rich phenomena displayed by the CSA potential by disentangling the CSA potential and selecting the hard sphere repulsion plus the soft repulsion for single investigation, the present report is a first step towards the aim.

\section{ACKNOWLEDGEMENT}

Project supported by The National Natural Science Foundation of China (20673150).

\section{REFERENCES}

[1] Alder BJ, Wainwright TE. Phase transition for a hard sphere system. J Chem Phys 1957; 27: 1208.
[2] Wood WW, Jacobson JD. Preliminary results from a recalculation of the Monte Carlo equation of state of hard spheres. J Chem Phys 1957; 27: 1207.

[3] Hansen JP, McDonald IR. Theory of Simple Liquids, 2nd ed. Academic: New York; 1986.

[4] Ryzhov VN, Stishov SM. Repulsive step potential: A model for a liquid-liquid phase transition. Phys Rev E 2003; 67: 010201(R).

[5] Fomin YD, Ryzhov VN, Tareyeva EE. Generalized van der Waals theory of liquid-liquid phase transitions. Phys Rev E 2006; 74: 041201.

[6] Zhou S. Performance Evaluation of third-order thermodynamic perturbation theory and comparison with existing liquid state theories. J Phys Chem B 2007; 111: 10736.

[7] Skibinsky A, Buldyrev SV, Franzese G, Malescio G, Stanley HE. Liquid-liquid phase transitions for soft-core attractive potentials. Phys Rev E 2004; 69: 061206.

[8] Zhou S. Thermodynamic perturbation theory in fluid statistical mechanics. Phys Rev E 2006; 74: 031119.

[9] Carnahan NF, Starling KE. Equation of state for nonattracting rigid spheres. J Chem Phys 1969; 51: 635.

[10] Franzese G, Malescio G, Skibinsky A, Buldyrev SV, Stanley HE. Generic mechanism for generating a liquid-liquid phase transition. Nature (London) 2001; 409: 692.

[11] Jagla EA. Core-softened potentials and the anomalous properties of water. J Chem Phys 1999; 111: 8980.

[12] Netz PA, Buldyrev SV, Barbosa MC, Stanley HE. Thermodynamic and dynamic anomalies for dumbbell molecules interacting with a repulsive ramplike potential. Phys Rev E 2006; 73: 061504.

[13] de Oliveira AB, Netz PA, Colla T, Barbosa MC. Thermodynamic and dynamic anomalies for a three-dimensional isotropic core-softened potential. J Chem Phys 2006; 124: 084505.

[14] Caballero JB, Puertas AM. Density anomaly and liquid-liquid transition from perturbation theories. Phys Rev E 2006; 74: 051506.

[15] Katayama Y, Mizutani T, Utsumi W, Shimomura O, Yamakata M, Funakoshi K-I. A first-order liquid-liquid phase transition in phosphorous. Nature (London) 2000; 403: 170.

[16] Giovambattista N, Stanley HE, Sciortino F. Relation between the high density phase and the very-high density phase of amorphous solid water. Phys Rev Lett 2005; 94: 107803.

[17] Mitus AC, Patashinski AZ. The liquid-liquid phase transition. Phys Lett A 1985; 113: 41

[18] Poole PH, Sciortino F, Essmann U, Stanley HE. Phase behaviour of metastable water. Nature (London) 1992; 360: 324

[19] Zhou S. Solid-liquid phase transition of the hard-core attractive Yukawa system and its colloidal implication. J Phys Chem B 2004; 108: 8447.

[20] Tanaka S, Yamamoto M, Ito K, Hayakawa R, Ataka M. Relation between the phase separation and the crystallization in protein solutions. Phys Rev E 1997; 56: R67.

[21] Ten Wolde PR, Frenkel D. Enhancement of protein crystal nucleation by critical density fluctuations. Science 1997; 277: 1975.

[22] Talanquer V, Oxtoby D. Crystal nucleation in the presence of a metastable critical point. J Chem Phys 1998; 109: 223.

[23] Lacks DJ. First-order amorphous-amorphous transformation in silica. Phys Rev Lett 2000; 84: 4629.

[24] Wilding MC, Benmore CJ, McMillan PF. A neutron diffraction study of yttrium- and lanthanum-aluminate glasses. J Non-Cryst Solids 2002; $297: 143$.

[25] Mishima O, Stanley HE. Decompression-induced melting of ice IV and the liquid-liquid transition in water. Nature 1998; 392: 164.

[26] Gibson HM, Wilding NB. Metastable liquid-liquid coexistence and density anomalies in a core-softened fluid. Phys Rev E 2006; 73: 061507.

[27] Tanaka H. General view of a liquid-liquid phase transition. Phys Rev E 2000; 62: 6968 .

[28] de Oliveira AB, Netz PA, Colla T, Barbosa MC. Structural anomalies for a three dimensional isotropic core-softened potential. J Chem Phys 2006; 125: 124503 . 